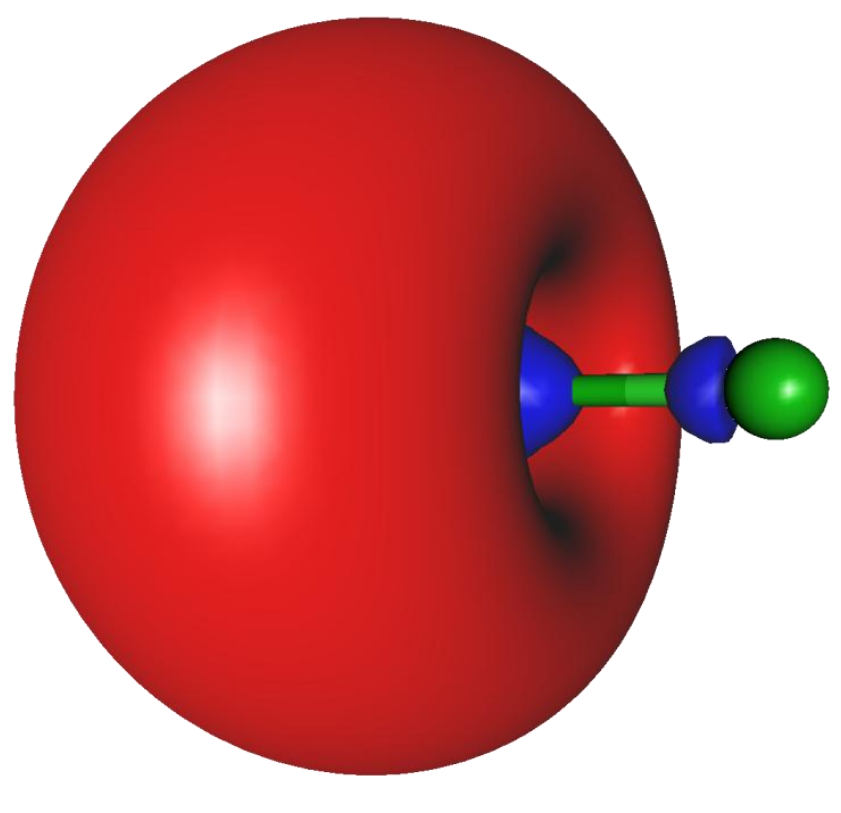

$\mathrm{BaCl}$

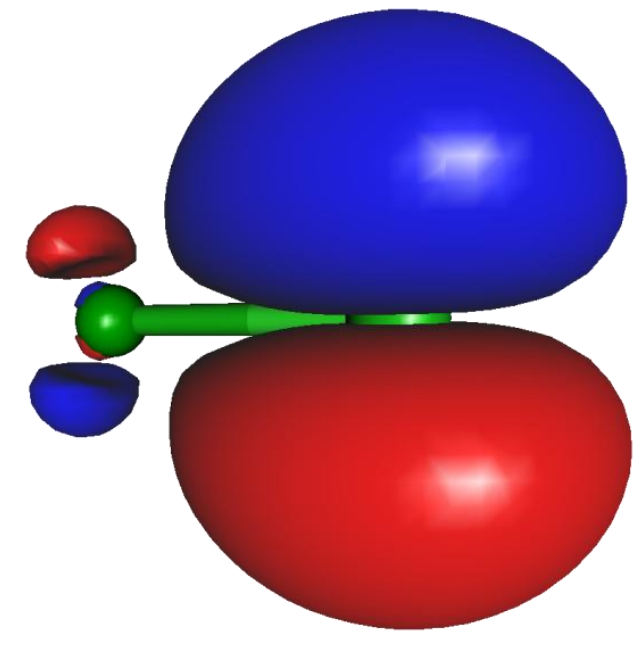

$\mathrm{BaCl}^{+}$ 


\title{
Characterization of the $\mathrm{BaCl}^{+}\left(\mathrm{X}^{1} \Sigma^{+}\right)$cation by photoelectron spectroscopy
}

Joshua H. Bartlett, Robert A. VanGundy and Michael C. Heaven*

Department of Chemistry, Emory University, Atlanta, GA 30322

\begin{abstract}
The $\mathrm{BaCl}^{+}$cation is of interest as it has the potential for being cooled to ultra-cold temperatures. Recent experiments have demonstrated sympathetic cooling of $\mathrm{BaCl}^{+}$ (Rellergert et al. Nature, 495 (2013) 490), but characterization of the internal state population distributions is hampered by the lack of spectroscopic data for this ion. In the present study, pulsed field ionization -zero kinetic energy photoelectron spectroscopy has been used to observe the $v^{+}=0-3$ vibrational levels of $\mathrm{BaCl}^{+}\left(\mathrm{X}^{1} \Sigma^{+}\right)$. Vibrational constants and an accurate value for the ionization energy of $\mathrm{BaCl}$ are reported. Comparisons to the predictions of high-level electronic structure calculations are also provided.
\end{abstract}

*Corresponding author. E-mail: mheaven@emory.edu, Phone: 404727 6617, FAX 404 7276586 


\section{Introduction}

Diatomic cations of the form $\mathrm{MX}^{+}$, where $\mathrm{M}$ is an alkaline earth metal and $\mathrm{X}$ is a halogen, are of interest for studies of molecular physics and chemistry at low temperatures[1-6]. The advantage of using ions is that they can be readily trapped using external fields. The $\mathrm{M}^{+}$ions are well suited for laser cooling as they have transitions that are analogous to those of the alkali metals. Coulomb crystals of $\mathrm{M}^{+}$are readily formed in rf traps. Reactions of these atomic ions yield diatomic products that are sympathetically cooled to low translational temperatures by the surrounding atomic ions. In addition to using laser ablation as an ion source, the molecular ions $\mathrm{BaF}^{+}, \mathrm{BaCl}^{+}$and $\mathrm{BaO}^{+}$have been generated by the reactions of laser cooled $\mathrm{Ba}^{+}$with $\mathrm{SF}_{6}, \mathrm{CH}_{3} \mathrm{Cl}$, and $\mathrm{O}_{2}$, respectively[3, 7]. As discussed by Hudson[4], the long-range repulsive forces between $\mathrm{M}^{+} / \mathrm{MX}^{+}$pairs mediate translational cooling, but they are not effective in cooling the internal degrees of freedom. The short-range interactions between an $\mathrm{MX}^{+}$ion and a laser cooled neutral atom will be far more effective in achieving ro-vibrational relaxation. This brings to light another advantage of the $\mathrm{MX}^{+}$species. They are closed-shell ions that can interact nonreactively with a range of neutral atoms. As an example, Hudson and co-workers have demonstrated vibrational cooling of $\mathrm{BaCl}^{+}$induced by interactions with laser-cooled $\mathrm{Ca}$ atoms $[1,2]$. This is a milestone achievement, but the study also highlights one of the current problems encountered in working with the diatomic ions of interest. There are almost no gas phase spectroscopic data for these species, and this information is essential for the characterization of internal state population distributions. To obtain the vibrational state distributions for $\mathrm{BaCl}^{+}$, Hudson and co-workers [1, 2] analyzed the band contours for the free-bound ${ }^{1} \Pi-X^{1} \Sigma^{+}$transition, which was detected by monitoring the 
rate of $\mathrm{Ba}^{+}$ion production. $\mathrm{Ab}$ initio electronic structure calculations were used to guide the interpretation of the photodissociation spectrum.

In the present study we have used pulsed-field ionization, zero-kinetic energy photoelectron (PFI-ZEKE) spectroscopy to observe the low-energy $\left(v^{+}=0-3\right)$ of $\mathrm{BaCl}^{+}$. These measurements validate the ground state potential energy curve used to analyze the photofragmentation data of Chen et al.[2], and provide an accurate ionization energy (IE) for $\mathrm{BaCl}$. The results are also used to assess the predictive capabilities of various electronic structure calculations.

\section{Experimental}

The apparatus used for these measurements has been described previously[8]. $\mathrm{BaCl}$ was produced in the gas phase by pulsed laser vaporization of $\mathrm{Ba}$ in the presence of a $\mathrm{He} / \mathrm{Cl}_{2}$ gas mixture $\left(0.1 \% \mathrm{Cl}_{2}\right)$. The gas mixture was subject to supersonic expansion, in order to cool the reaction products. The reagents used consisted of natural abundance isotopic mixtures for both $\mathrm{Ba}$ and $\mathrm{Cl}$. Isotope shifts were below the resolution of these measurements. Two-color photoionization was used to examine the low energy states of $\mathrm{BaCl}^{+}$. This was accomplished using two independently tunable pulsed dye lasers (with linewidths of 0.15 and $0.3 \mathrm{~cm}^{-1}$, FWHM). The first laser pulse was tuned to excite the unresolved Q-branch feature of the $\mathrm{BaCl}, \mathrm{C}^{2} \Pi_{3 / 2}-\mathrm{X}^{2} \Sigma^{+}, 0-0$ band at $19453.0 \mathrm{~cm}^{-1}$ [9]. Laser induced fluorescence spectroscopy of the $\mathrm{C}-\mathrm{X}$ transition was used to optimize the tuning of the first laser. Once this resonance had been established, the second laser was tuned over the wavelength range where the sum of the photon energies would span the

ionization threshold. Previous studies indicated an IE of 40,000 $\pm 400 \mathrm{~cm}^{-1}$ (see [10] and 
references therein). A time-of-flight mass spectrometer was used to monitor the flux of ${ }^{138} \mathrm{Ba}^{35} \mathrm{Cl}^{+}$ions, thereby producing a photoionization efficiency spectrum. This scan yielded a preliminary IE of $40,540 \pm 15 \mathrm{~cm}^{-1}$. The IE was further refined by recording the PFI-ZEKE spectrum. For these measurements the dye lasers were used to excite $\mathrm{BaCl}$ to long-lived, high-n Rydberg states that lie just below the ionization threshold. This was done under field-free conditions. After a delay of $2 \mu$ s a pulsed field of $0.36 \mathrm{~V} / \mathrm{cm}$ was applied to ionize the excited $\mathrm{BaCl}$, and convey the electrons to a microchannel plate detector. The absolute wavenumber calibration of the second dye laser was established by recording resonantly enhanced one-color, three-photon $(1+2)$ ionization of $U$. The $U$ atomic line positions were obtained from the NIST atomic spectra database[11].

\section{Results and Discussion}

PFI-ZEKE measurements were used to observe the $v^{+}=0-3$ vibrational levels of the $\mathrm{BaCl}^{+}$ground state. Fig. 1 shows a composite scan over these features. The PFIZEKE scans did not yield resolvable rotational structure. Consequently, we have used the maximum intensity points to determine the band positions, with an estimated relative uncertainty of $\pm 1 \mathrm{~cm}^{-1}$. The band energies, relative to $\mathrm{BaCl} \mathrm{X} \mathrm{X}^{+}, \mathrm{v}=0$, are listed in Table 1. Due to the fact that the rotational structure was not resolved, we estimate that the absolute uncertainty for the IE is $\pm 5 \mathrm{~cm}^{-1}$. Fitting the Morse vibrational energy expression,

$$
\mathrm{G}\left(v^{+}\right)=\omega_{\mathrm{e}}\left(v^{+}+1 / 2\right)-\omega_{\mathrm{e}} \mathrm{x}_{\mathrm{e}}\left(v^{+}+1 / 2\right)^{2}
$$

to the band energies yielded vibrational constants of $\omega_{\mathrm{e}}=342(2)$ and $\omega_{\mathrm{e}} \mathrm{X}_{\mathrm{e}}=1.6(4) \mathrm{cm}^{-1}$. 
Electronic structure calculations were used to predict the ground state molecular constants of $\mathrm{BaCl}$ and $\mathrm{BaCl}^{+}$, and the $\mathrm{IE}$ of $\mathrm{BaCl}$. These calculations were performed using the Molcas 7.8 suite of programs[12]. The triple zeta ANO-RCC-VTZP basis sets were used for both $\mathrm{Ba}$ and $\mathrm{Cl}$. Scalar relativistic effects were included by using the second order corrections of the Douglas-Kroll-Hess Hamiltonian. Single point energies, spaced by $0.02 \AA$, were calculated over the internuclear distance range from 2.3 to $3.5 \AA$. Vibrational constants and the equilibrium bond lengths were obtained by fitting the lower energy points ( $\mathrm{E}<5000 \mathrm{~cm}^{-1}$, relative to the potential minimum) to a Morse potential. The predictions from B3LYP density functional theory, CASPT2 and CCSD(T) calculations are compared with the experimental results in Table 2. This Table also includes the results of the CASPT2 calculations reported by Chen et al.[2] in their study of $\mathrm{BaCl}^{+}$ photodissociation. All of the methods examined gave reasonable predictions for both $\mathrm{BaCl}$ and $\mathrm{BaCl}^{+}$(Table 2 presents the adiabatic IE values from the calculations). Note that all of the methods slightly underestimated the harmonic vibrational frequencies. The good agreement between the CASPT2 calculations of Chen et al.[2] and the experimental data provide additional evidence in support of their interpretation of the $\mathrm{BaCl}^{+}$free-bound spectrum.

The present theoretical results for the IE are also respectable, indicating that the calculations correctly reproduce the increase in the bond energy that accompanies ionization. The reason for this increase in the bond energy, contraction of the bond length, and increase in the vibrational frequency is easily understood by examination of the highest occupied molecular orbitals (HOMO) of $\mathrm{BaCl}$ and $\mathrm{BaCl}^{+}$. The singly occupied $\mathrm{HOMO}$ of $\mathrm{BaCl}$ is shown on the right hand side of Fig. 2. This orbital is a 
metal centered $6 s / 6 p$ hybrid that is polarized away from the negatively charged $\mathrm{Cl}$. It is somewhat anti-bonding in character. The $\mathrm{HOMO}$ for $\mathrm{BaCl}^{+}$is composed primarily of the $\mathrm{Cl} 3 p \pi$ orbitals ( $3 p_{x}$ is shown in Fig. 2). Hence, ionization of $\mathrm{BaCl}$ removes the $6 s$ electron and reduces the electrostatic repulsion. This well defined ionic character also explains why the ground state properties of both $\mathrm{BaCl}$ and $\mathrm{BaCl}^{+}$are readily predicted using single-reference electronic structure methods.

Lastly, it is of interest to compare the vibrational constant for $\mathrm{BaCl}^{+}(\mathrm{X})$ with the constants for the more highly excited states of $\mathrm{BaCl}$. The E, F, and $\mathrm{G}$ states (all ${ }^{2} \Sigma^{+}$), with term energies of $27064.8,29493.6$ and $32511.4 \mathrm{~cm}^{-1}$, have harmonic vibrational frequencies of $311.5,331.8$ and $331.3 \mathrm{~cm}^{-1}$ [13]. This trend suggests that the vibrational constants are converging toward the value for the ion as the energy increases, as would be expected for a series of Rydberg states.

\section{Acknowledgements}

We thank Prof. Laura Gagliardi (University of Minnesota) and Dr. Ignacio Fernández Galván (Uppsala University, Sweden) for advice concerning relativistic calculations for $\mathrm{BaCl}^{+}$. This work was supported by the National Science Foundation under grant CHE-1265586. The electronic structure calculations reported here were carried out using the resources of the Cherry Emerson Center for Scientific Computation, which is supported by the NSF MRI-R2 grant CHE-0958205. 


\section{References}

[1] W.G. Rellergert, S.T. Sullivan, S.J. Schowalter, S. Kotochigova, K. Chen, E.R.

Hudson, Nature (London, U. K.), 495 (2013) 490-494.

[2] K. Chen, S.J. Schowalter, S. Kotochigova, A. Petrov, W.G. Rellergert, S.T. Sullivan,

E.R. Hudson, Phys. Rev. A At., Mol., Opt. Phys., 83 (2011) 030501/030501030501/030504.

[3] M.V. DePalatis, M.S. Chapman, Phys. Rev. A At., Mol., Opt. Phys., 88 (2013) 023403/023401-023403/023407.

[4] E.R. Hudson, Phys. Rev. A At., Mol., Opt. Phys., 79 (2009) 032716/032711032716/032719.

[5] P. Puri, S.J. Schowalter, S. Kotochigova, A. Petrov, E.R. Hudson, J. Chem. Phys., 141 (2014) 014309/014301-014309/014305.

[6] J.H.V. Nguyen, C.R. Viteri, E.G. Hohenstein, C.D. Sherrill, K.R. Brown, B. Odom, New J. Phys., 13 (2011) 063023/063021-063023/063028.

[7] J.E. Goeders, C.R. Clark, G. Vittorini, K. Wright, C.R. Viteri, K.R. Brown, J. Phys. Chem. A, 117 (2013) 9725-9731.

[8] M.C. Heaven, B.J. Barker, I.O. Antonov, J. Phys. Chem. 118 (2014) 10867-10881.

[9] P. Pages, A. Pereira, P. Royen, Phys. Scr., 31 (1985) 281-285.

[10] L.A. Kaledin, M.C. Heaven, R.W. Field, J. Mol. Spectrosc., 193 (1999) 285-292.

[11] Kramida, A., Ralchenko, Yu., Reader, J., and NIST ASD Team (2014). NIST Atomic Spectra Database (ver. 5.2), [Online]. Available: http://physics.nist.gov/asd [2015, July 20]. NIST, Gaithersburg, MD. 
[12] F. Aquilante, L. De Vico, N. Ferre, G. Ghigo, P.-a. Malmqvist, P. Neogrady, T.B.

Pedersen, M. Pitonak, M. Reiher, B.O. Roos, L. Serrano-Andres, M. Urban, V. Veryazov, R. Lindh, J. Comp. Chem., 31 (2010) 224-247.

[13] K.P. Huber, G. Herzberg, Molecular Spectra and Molecular Structure, 4: Constants of Diatomic Molecules, 1979.

[14] P. Pages, A. Pereira, P. Royen, Phys. Scr., 31 (1985) 281-285. 
Table 1. Band energies and vibrational intervals for $\mathrm{BaCl}^{+} \mathrm{X}^{1} \Sigma^{+}$

$\begin{array}{ccc}v^{+} & \mathrm{E}^{a} / \mathrm{cm}^{-1} & \Delta \mathrm{E} / \mathrm{cm}^{-1} \\ 0 & 40549.2 & \\ 1 & 40888.3 & 339.1 \\ 2 & 41222.3 & 334.0 \\ 3 & 41555.0 & 332.7\end{array}$

a. The band energies are relative to $\mathrm{BaCl} \mathrm{X}^{2} \Sigma^{+}, v=0$.

Table 2. Calculated and measured spectroscopic constants for $\mathrm{BaCl}$ and $\mathrm{BaCl}^{+}$

$\begin{array}{ccccc}\text { Method } & \omega_{\mathrm{e}} / \mathrm{cm}^{-1} & { }^{138} \mathrm{Ba}^{35} \mathrm{Cl}^{+} \mathrm{X}^{1} \Sigma^{+} \\ \omega_{\mathrm{e} X} / \mathrm{cm}^{-1} & \mathrm{R}_{\mathrm{e}} / \AA & \mathrm{IE} / \mathrm{cm}^{-1} \\ \text { CASPT2 }^{a} & 328.3 & 1.56 & 2.57 & \\ \text { CASPT2 } & 332.3 & 0.79 & 2.61 & \\ \text { CCSD(T) } & 327.9 & 0.82 & 2.61 & \\ \text { B3LYP/DFT } & 323.7 & 0.81 & 2.62 & \\ & & & & \\ \text { Expt }^{b} & 342(2) & 1.6(4) & - & \\ & & & & \\ \text { CASPT2 } & 273.3 & 0.90 & 2.72 & 40170 \\ \text { CCSD(T) } & 271.4 & 0.87 & 2.72 & 40436 \\ \text { B3LYP/DFT } & 268.5 & 0.89 & 2.72 & 42021 \\ \text { Exp. } & 279.9^{c} & 0.90^{c} & 2.68^{c} & 40549^{b}\end{array}$

a. Chen et al.[2]

b. This work.

c. Constant from ref. [14]. The $\mathrm{R}_{\mathrm{e}}$ value was calculated from the equilibrium rotational constant, $\mathrm{B}_{\mathrm{e}}=0.0839673 \mathrm{~cm}^{-1}$. 


\section{Figure captions}

1. PFI-ZEKE spectra showing the vibrational levels of $\mathrm{BaCl}^{+}\left(\mathrm{X}^{1} \Sigma^{+}\right)$. The energy scale is relative to $\mathrm{BaCl}\left(\mathrm{X}^{2} \Sigma^{+}\right) v^{\prime \prime}=0$. Note that the vertical scale for the $v^{+}=3$ band has been expanded by a factor of five.

2. The highest energy occupied molecular orbitals for $\mathrm{BaCl}$ (left) and $\mathrm{BaCl}^{+}$(right).

These plots were generated using an isovalue of 0.02 . 


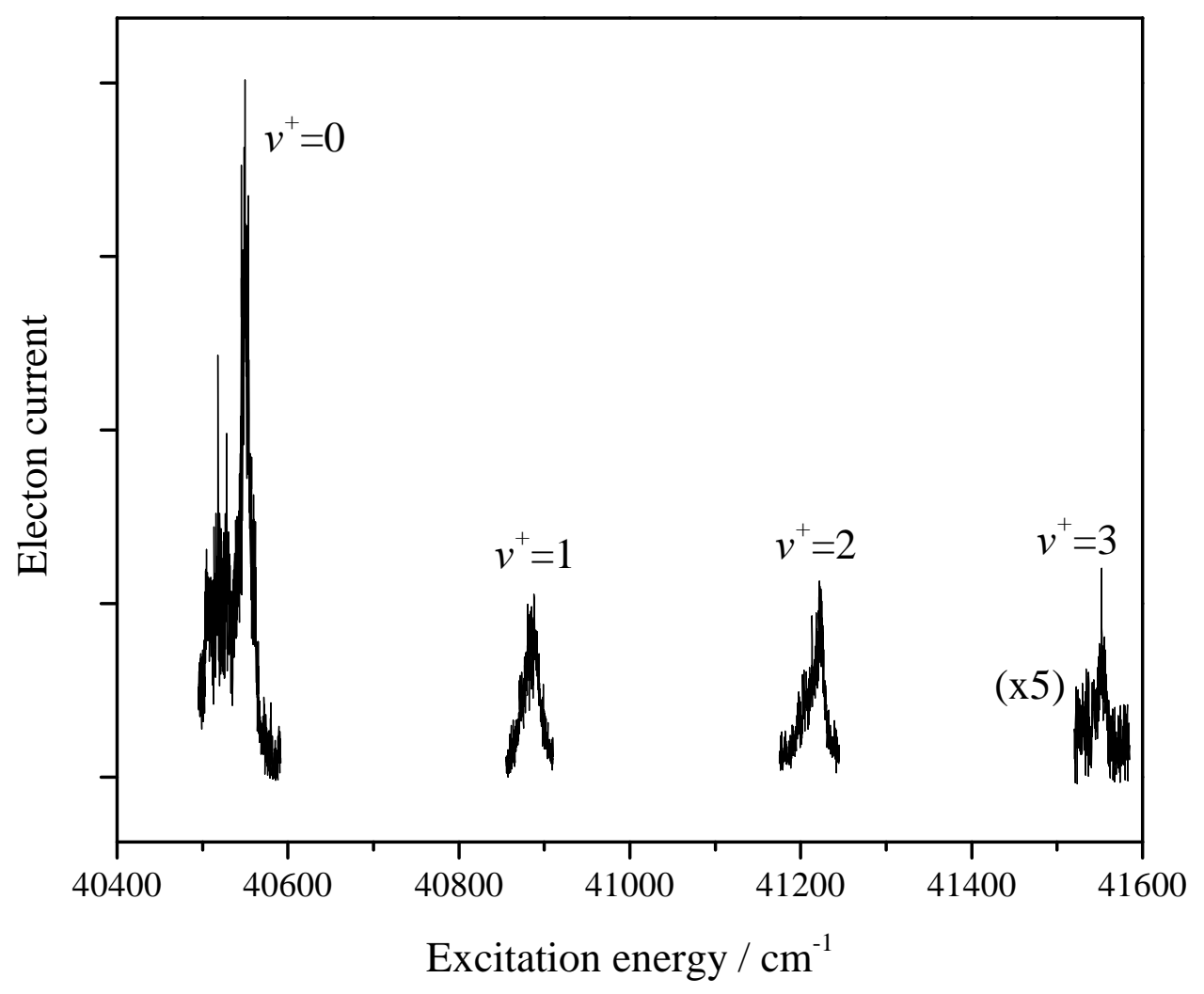

Fig. 1 


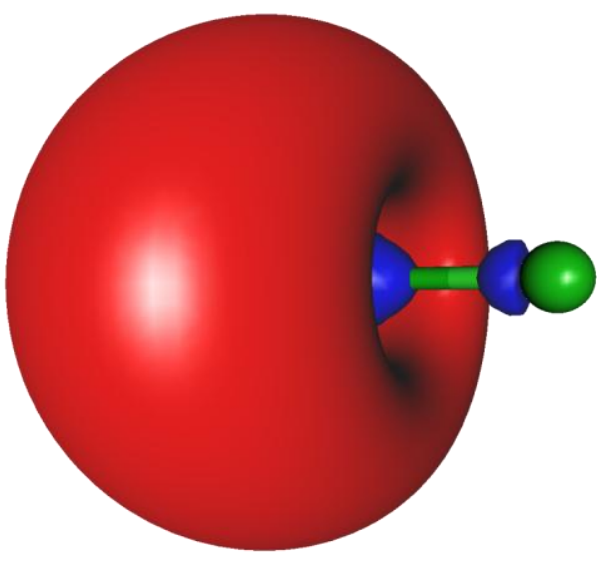

$\mathrm{BaCl}$

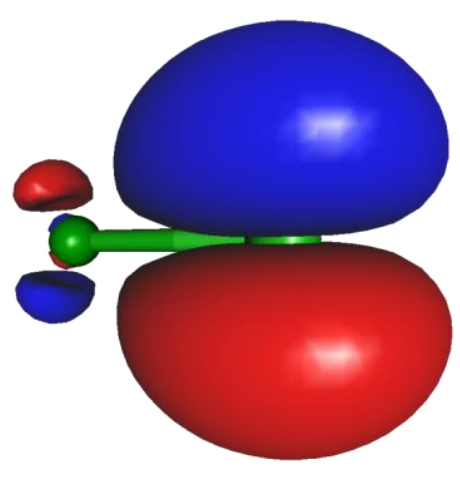

$\mathrm{BaCl}^{+}$

Fig. 2 